\title{
E Editorial
}

El ser humano, permanente en la búsqueda del confort, ha emprendido en el desarrollo de una variedad de tecnologías que aportan de manera significativa a mejorar su calidad de vida; esta condición ha provocado que muchos de los procesos naturales se vean afectados y que la armonía hombre naturaleza sufra perturbaciones.

Un accionar responsable y consecuente con la sociedad y el ambiente ha ocasionado que el interés de investigadores se centre en buscar armonizar el desarrollo tecnológico con la sostenibilidad del planeta; esta ardua tarea en la que la Universidad y sus actores deben participar y desde sus claustros promover la incorporación de innovaciones que aporten al objetivo planteado es un compromiso de todos.

En este número INGENIUS presenta el análisis en lo referente a Control, Generación y Distribución de la Energía Eléctrica, la Aplicación de Sistemas SCADA, orientado a la aplicación de redes; se analizan algunos componentes como el transformador en el cual mediante la aplicación de técnicas innovadoras se detectan las fallas en el mismo y se puede conseguir un sistema eficiente sin perdidas, lo que aporta de manera significativa a la reducción de costos de mantenimiento y la disminución de contaminación, a esto se suma un análisis desde el punto de vista del consumidor, en donde se exponen planes de ahorro de energía que beneficiarán tanto al consumidor como al cuidado del medio ambiente.
El Etiquetado de Roles Semánticos es un tema crucial en el tratamiento automático del lenguaje natural, la experiencia aquí presentada nos permite contextualizar el alcance de la aplicación de modelos de máxima entropía en este proceso.

Otro aspecto que se analiza es la contaminación provocada por los aceites de los vehículos; el estudio considera la posibilidad de aumentar la durabilidad de uso de este producto y con esto reducir la producción de este contaminante responsable de un daño significativo en las urbes de nuestro país. También se aborda el desarrollo de la inteligencia acompañado de la música de Mozart, sus efectos y connotaciones en los seres humanos, en donde el arte se analiza desde el mundo de la ciencia.

Finalmente el desarrollo científico y tecnológico han aportado de manera significativa a la globalización esto se puede evidenciar en lo referente al comercio electrónico; esta revisión del estado del arte aporta de manera significativa al conocimiento y entendimiento de esta tendencia.

La variedad en las temáticas de los artículos científicos en este número hace que INGENIUS se convierta en la ventana que permite mostrar a la sociedad el arduo y comprometido trabajo de muchos investigadores con la sociedad.

Ing. John Calle S. M.Sc. Editor responsable 